\title{
Tratamiento quirúrgico vs terapia periodontal básica: estudios longitudinales en periodoncia clínica
}

\author{
FABRIZI S* \\ BARBIERI PETRELLI G** \\ VIGNOLETTI $\mathbf{F} * *$ \\ BASCONES-MARTÍNEZ A***
}

\begin{abstract}
Fabrizi S, Barbieri Petrelli G, Vignoletti F, Bascones Martínez A. Tratamiento quirúrgico vs terapia periodontal básica: estudios longitudinales en periodoncia clínica. Av Periodon Implantol. 2007; 19, 2: 161-175.
\end{abstract}

\begin{abstract}
RESUMEN
Las enfermedades periodontales son unas graves infecciones bacterianas que destruyen las fibras de inserción y el hueso de soporte que mantienen los dientes en la boca. Sin tratar, esta enfermedad puede llevar a la pérdida dental (Medical Dictionary). Los estudios longitudinales han centrado su atención hacia la periodontitis crónica. Se ha documentado el decisivo papel de la placa bacteriana en la iniciación y en el mantenimiento de la gingivitis, y que, los efectos dañinos sobre los tejidos y la gravedad de estos efectos están regulados por una compleja interacción entre el parásito y huésped. El tratamiento de la lesión periodontal cumple, para el tratamiento periodontal básico, con el propósito de eliminar y prevenir la recurrencia de los depósitos bacterianos localizados en las superficies dentarias supragingivales y subgingivales y, para el tratamiento quirúrgico con el objetivo de crear acceso para el desbridamiento profesional correcto de las superficies radiculares infectadas y establecer una morfología gingival adecuada que facilite el autocontrol de la placa por parte del paciente. Diferentes técnicas se han utilizado para alcanzar el objetivo de mejorar el pronóstico de los dientes a largo plazo. Desafortunadamente no son muchos los estudios que consiguen demostrar la efectividad de las técnicas utilizadas, con una evolución a lo largo del tiempo dejando entonces algunas incertidumbres.
\end{abstract}

\section{PALABRAS CLAVE}

Terapia/enfermedad periodontal, terapia no-quirúrgica, terapia quirúrgica; revisión sistemática.

Fecha de recepción: Febrero 2007.

Aceptado para publicación: Abril 2007.

\section{INTRODUCCIÓN}

La enfermedad periodontal es una grave infección bacteriana que destruye las fibras de inserción y el hueso de soporte que mantienen los dientes en la boca. Sin tratar, esta enfermedad puede llevar a la pérdida dental (Medical Dictionary).
Entre las distintas formas de enfermedades periodontales, los estudios con seguimiento a largo plazo, hoy en día disponibles, centran su atención hacia una en particular, la periodontitis crónica. Esta es definida como una enfermedad inflamatoria de los tejidos de soporte de los dientes causada por un grupo de microorganismos específicos, resultante en una destruc- 
ción progresiva del ligamento periodontal y del hueso alveolar, con formación de una bolsa periodontal, recesión o ambas (1). Ésta puede clasificarse en base a su extensión (localizada y generalizada) y a su severidad (leve, moderada y severa) y además puede asociarse con factores modificantes como las enfermedades sistémicas, tabaco y factores locales.

Estudios epidemiológicos transversales indican que aproximadamente el $10-15 \%$ de la población adulta sufre una "periodontitis avanzada" mientras que el $80 \%$ tiene una "periodontitis moderada" y el $10 \%$ de la población es periodontalmente sana (2).

Aunque se haya documentado el decisivo papel de la placa bacteriana en la iniciación y en el mantenimiento de la gingivitis, no obstante estudios de asociación demostraron la estrecha relación entre la presencia de la placa bacteriana y el desarrollo de la "enfermedad periodontal", estos autores afirman que hay un mayor número de microorganismos patógenos que pueden aislarse en las lesiones periodontales en comparación con aquellos aislados en las localizaciones sanas) (3-8), los efectos dañinos sobre los tejidos y la gravedad de estos efectos están regulados por una compleja interacción entre el parásito y huésped. (9)

El tratamiento de la lesión periodontal cumple, para el tratamiento periodontal básico, con el propósito de eliminar y prevenir la recurrencia de los depósitos bacterianos localizados en las superficies dentarias supragingivales y subgingivales y, para el tratamiento quirúrgico con el objetivo de crear acceso para el desbridamiento profesional correcto de las superficies radiculares infectadas y establecer una morfología gingival adecuada que facilite el autocontrol de la placa por parte del paciente, pretendiendo así mejorar el pronóstico de sus dientes a largo plazo.

Los parámetros hoy en día utilizados como criterios de evaluación del estado periodontal, es decir, los cambios en la profundidad de sondaje (PS), los cambios en el nivel clínico de inserción (NI) y las alteraciones en altura del hueso y de los tejidos marginales gingivales, aunque no nos permitan evaluar exactamente el estado efectivo de las localizaciones analizadas, por cierto ayudan a evaluar los efectos posteriores a la enfermedad y de alguna manera pronosticar un eventual riesgo de recurrencia. Signos como la progresiva pérdida de inserción, el sangrado al sondaje y el progresivo aumento de la movilidad son patognomónicos de un estado activo de la enfer- medad o de una mayor posibilidad de recurrencia de la misma. Resultados provenientes de algunos estudios $(10,11)$ demuestran que los sujetos con un elevado porcentaje de bolsas residuales con $\mathrm{PS} \geq 6 \mathrm{~mm}$ tras el tratamiento, corrían un mayor riesgo de padecer una pérdida adicional de inserción con respecto a los sujetos con un porcentaje de bolsas profundas residuales menor.

Tanto la reducción de la profundidad de bolsa (PB) como el mantenimiento del estado de salud (no inflamación), y la ganancia del tejido de soporte para los dientes (ganancia de inserción) son los principales objetivos clínico de la terapia periodontal.

Diferentes técnicas se han utilizado para alcanzar este objetivo. Desafortunadamente no son muchos los estudios que consiguen demostrar la efectividad de las técnicas utilizadas, con una evolución a lo largo del tiempo faltando entonces claridad en este tema.

Por definición, solamente los estudios longitudinales nos proporcionan la capacidad, mediante el seguimiento a largo plazo, discriminar los efectos de un determinado tratamiento y la capacidad de afirmar en qué caso uno es mejor que otro.

Los estudios que presentaremos a continuación son algunos de los estudios longitudinales hoy en día disponibles en la literatura científica, que han hecho la historia de la periodoncia clínica y que han puesto las bases para el actual conocimiento de los efectos de la terapia periodontal. Dividiremos éstos, según las escuelas de origen de los autores que corresponde también a las diferentes escuelas de pensamiento.

\section{ESCUELA DE MICHIGAN}

\section{MiChIGAN I}

Ramfjord fue el primero en efectuar un ensayo clínico randomizado y controlado con seguimiento a largo plazo de los resultados de las terapias periodontales. Este estudio publicado en 1968 informó de los datos recogidos durante un seguimiento de 6 años desde 1961 a 1966.

Después, en el año 1973 publicó, junto con sus colaboradores, el estudio definitivo que reportaba los datos precedentes, más los nuevos recogidos hasta 1972 evaluando cirugía de eliminación de bolsa frente al curetaje subgingival (12). 
Basándose en el concepto clínico que la bolsa periodontal es un defecto autoperpetuante capaz, antes o después, de desarrollar una ulterior perdida de inserción, afirmaban la necesidad de su eliminación para conseguir la curación de la "periodontitis". La evaluación de los resultados de la terapia periodontal estaba basada en un reexamen de la profundidad del "surco" en varios intervalos de tiempo después de la terapia, con la implicación que si el surco llegara a una profundidad de sondaje superior a 3-4 $\mathrm{mm}$ el resultado de la terapia seria insatisfactorio.

Con los objetivos de (1) averiguar, en función del NI, cual de los tratamientos periodontales conseguía mejores resultados a largo plazo, (2) probar la eficacia de estos tratamientos en la eliminación de las bolsas periodontales a largo plazo e (3) investigar si la eliminación de la bolsa periodontal es necesaria para el mantenimiento a largo plazo de la inserción periodontal estudiaron una población de 104 sujetos con un total de 2604 dientes (53 perdidos durante el estudio).

Tras el análisis de la historia médica, de la toma de fotografías a colores y examen radiográfico, analizaron los "niveles de gingivitis", placa y cálculo, la profundidad de sondaje y el nivel clínico de inserción tomando como unidad de estudio las localizaciones (Mesial, Distal, Vestibular y Lingual) de todos los dientes. Estas medidas siempre la efectuaba el mismo examinador para cada paciente. Había tres examinadores y los tres estaban calibrados y ciegos a las mediciones precedentes.

Tras la toma de los datos al inicio, todos los pacientes recibieron rapado y alisado radicular (RAR), instrucciones de higiene oral (IHO) y ajuste oclusal. Los pacientes del grupo experimental se trataron cada uno con cirugía de eliminación de bolsa en un lado y curetaje subgingival en el contralateral (diseño de boca partida). Una higienista realizó cada 3 meses profilaxis e IHO. Los registros se tomaron una vez al año.

Las variables respuesta solo se compararon con los valores iniciales y se analizaron, según las 4 localizaciones/diente, la PS y el NI. El test de la $t$ de Student se utilizó para evaluar la significación estadística con un intervalo de confianza de $99 \%$. No existieron diferencias estadísticamente significativas entre los dos grupos en la visita inicial. Los resultados se analizaron también en función de la terapéutica utilizada.

En el cómputo global con los dos tratamientos, los resultados mostraron una pequeña ganancia de inser- ción durante el primer año (media de los valores de todas las localizaciones tras los dos tratamientos). Esta ganancia se fue perdiendo hasta llegar a ser estadísticamente significativa a los 4 años.

Así también fueron los resultados evaluando la profundidad de bolsa, que se vio reducida de manera estadísticamente significativa durante los primeros 6 años, aunque estas diferencias se perdieron sin llegar a los niveles iniciales al séptimo año.

En la comparación intergrupo también había diferencias estadísticamente significativas durante los primeros tres años donde, tras el RAR, se obtuvieron mejores resultados que con cirugía de eliminación de bolsa en cuanto a los cambios en el nivel de inserción. Desde el cuarto año todos perdieron inserción, siendo menores en el curetage.

En cuanto a los cambios en la profundidad de bolsa, se obtuvieron grandes mejoras con ambas técnicas (la más marcada el primer año y mejores resultados con la cirugía de eliminación de bolsa) pero luego las dos sufrieron regresión casi hasta los niveles iniciales.

\section{MICHIGAN II}

Este mismo grupo, en 1979, (13) efectuó un nuevo estudio siempre comparando distintas técnicas quirúrgicas (en este caso cirugía de eliminación de bolsa y el colgajo de Widman modificado (CWM) vs técnicas no quirúrgica (en este caso el "curetaje subgingival"). Ellos mismos criticaron la manera de reportar los resultados (la media de los valores de todos los dientes tratados y el análisis basado en la media de los valores de media boca) en su anterior articulo, donde no fue posible evaluar los cambios en cada bolsa en cuanto a las variaciones de profundidad de sondaje y nivel de inserción.

Así que, justifican este mismo concepto afirmando que "el pronóstico es un objetivo difícil de conseguir aunque de fundamental importancia en las consideraciones del plan de tratamiento y siendo la profundidad de sondaje y el nivel clínico de inserción tradicionalmente los elementos básicos por evaluar el pronóstico periodontal (cambios en los valores de PI y NI), se deben utilizar los valores iniciales de profundidad de sondaje y el nivel de inserción como puntos de referencias". Los objetivos de estos autores fueron (1) comparar los resultados de los tratamientos según la PS y (2) determinar si las modalidades de tratamiento in- 
fluyen en el mantenimiento de la inserción periodontal dependiendo de la severidad inicial de la enfermedad.

También para este estudio clínico randomizado, se utilizó el diseño de boca partida para tratar los 78 pacientes inicialmente incluidos (terminaron el estudio en 72 a 5 años y 43 a 8) con las diferentes técnicas: curetaje subgingival, cirugía con colgajo de Widman modificado y cirugía de eliminación de bolsa. Cada uno de los pacientes recibía 2 de estos tratamientos, 1 en cada hemimandíbula, y eran incluidos en un programa de mantenimiento cada tres meses llevado a cabo por una higienista.

Hicieron entonces, una estratificación de las bolsas periodontales en tres grupos diferentes:

\section{- Clase I: 1-3 mm de PS (bolsas no patológicas). \\ - Clase II: 4-6 mm de PS (bolsas moderadas). \\ - Clase III: 7-12 mm de PS (bolsas profundas).}

Las variables respuesta, PS y NI, fueron medidas por un periodoncista al inicio y cada año durante los ocho del estudio en las superficies vestibular, lingual, mesial y distal. Esta vez las unidades de análisis del estudio eran las localizaciones (las bolsas).

Los resultados, según esta estratificación demostraron, que la presencia de un elevado numero de "no bolsas" (1-3 mm) enmascararía los cambio relativos a las bolsas profundas (fenómeno de "regresión a la media"), al ser imposible reducir la PS o ganar inserción en surcos gingivales de 1-3 mm.

Con el objeto de solucionar este problema, se evaluaron los resultados exclusivamente para las bolsas verdaderas (Clase II y Clase III). Revelándose que la respuesta al tratamiento depende de la PB inicial y no de la localización en las superficies de los dientes.

Los resultados confirmaron los del estudio precedente viéndose reducidas las PS de manera estadísticamente significativa con los tres tratamientos y más específicamente para las bolsas de Clase II y Clase III, siendo la reducción significativamente mayor para las bolsas más profundas. Los resultados seguían las mismas direcciones al analizarla para la ganancia en el nivel de inserción.

También fue evaluada la variable modalidad de tratamiento. Los autores concluyeron que para las bolsas superficiales no hay que hacer tratamiento quirúrgico e intentar no incluir esta área en la cirugía de eliminación de las bolsas vecinas. En cuanto a la reducción de la PS en bolsas moderadas, esta era mayor con CWM (como consecuencia de la ganancia en el nivel de inserción y recesión del margen gingival libre) con respecto a la cirugía de eliminación de bolsa y al curetage. La ganancia del nivel de inserción fue significativa para los tres tratamientos durante los primeros tres años post operatorios y fueron estadísticamente significativos para el curetage subgingival y el CWM hasta los ocho años. A los 8 años solamente hubo diferencias estadísticamente significativas entre el curetage y la cirugía de eliminación de bolsa. En las bolsas profundas la terapia quirúrgica demostró ser más efectiva que el curetage, sin diferencias estadísticamente significativas entre el CWM y la cirugía de eliminación de bolsa mientras sí la había entre estas dos técnicas comparándolas según la ganancia del nivel clínico de inserción. En general los resultados mejores se obtuvieron con el CWM.

No se evaluaron los posibles efectos en los defectos de las furcaciones.

\section{MICHIGAN III}

Algunos años después Ramfjord y cols. en 1987 (15), continuando un estudio empezado por Hill y cols. 1981 (16), compararon los resultados de cuatro modalidades diferentes de tratamiento de la "enfermedad periodontal", con un grado de severidad de moderado a avanzado, en 90 sujetos.

Éstos fueron examinados inicialmente, se le realizó raspado y alisado radicular, y se les dieron instrucciones de higiene oral. De manera randomizada, a cada paciente se le efectuó un tratamiento en cada cuadrante. Los tratamientos evaluados fueron (1) cirugía de eliminación de con cirugía ósea (17); (2) cirugía con colgajo de Widman modificado (14); (3) curetage subgingival (18); (4) raspado y alisado radicular (18). La profilaxis post cirugía fue efectuada cada semana al principio y cada tres meses después. A los cinco años quedaron 72 pacientes.

Aunque estos autores no justificaron la necesidad de tratamiento para las bolsas de 1-3 mm (Clase I), los resultados reportados mostraron una pérdida de inserción gradual, al parecer como consecuencia del tratamiento, durante los cincos años, con menor pérdida de inserción tras el raspado y el curetage que tras los procedimientos quirúrgicos. 
Para las bolsas moderadas (Clase II) los dos tratamientos quirúrgicos obtuvieron mejores resultados tras el primer año con respecto al raspado y al curetage, obteniendo una mayor reducción de bolsa. Esta situación se mantuvo igual después de cinco años de seguimiento, con una significativa mayor reducción tras la cirugía de eliminación de bolsa que tras el curetage. Con respecto a la ganancia de inserción, resultó al principio ligeramente mayor para el RAR que con los dos tratamientos quirúrgicos, aunque todos los tratamientos conseguían ganancia de inserción. Se mantuvieron los resultados a los cincos años, aunque hubo una ligera pérdida de inserción para todos los tratamientos (mayor para la cirugía de eliminación de bolsa).

Las bolsas más profundas (Clase III) fueron reducidas de manera más pronunciada tras la cirugía de eliminación de bolsa y en menor cantidad tras el raspaje. No hubo diferencias estadísticamente significativas entre los diferentes tipos de tratamiento a los cincos años en la reducción de bolsa ni en los cambios del nivel clínico de inserción. Pero, a los cincos años, solamente el curetage y el raspaje mostraron una significativa ganancia de inserción clínica con respecto al inicio.

1. La respuesta al tratamiento depende de la PB inicial y no de la localización en las superficies de los dientes.

2. No se pueden tratar quirúrgicamente, ni con el RAR las bolsas superficiales (1-3 mm) por que es imposible reducir la profundidad de sondaje y además se provoca una pérdida gradual en el nivel de inserción. Mejores resultados, en cuanto a la pérdida de inserción, se obtienen con el raspaje y alisado radicular.

3. El curetage no aporta beneficio sobre el raspado y alisado radicular

4. En las bolsas moderadas, con el CWM se obtuvieron mejores resultados en cuanto a la reducción de la profundidad de sondaje con respecto a las otras técnicas.

5. En las bolsas moderadas hay una menor, aunque no ES, pérdida de inserción tras CWM que con cirugía de eliminación de bolsa.

6. Independientemente del tipo de tratamiento, el peor pronóstico es para los dientes con las lesiones periodontales que afectan las furcas.

7. En las bolsas profundas los resultados evaluados a largo plazo demuestran que los cuatro tratamientos son igualmente efectivos en cuanto a la reducción de bolsa y a los cambios en el nivel de inserción con respecto a los datos en basal.

\section{ESCUELA DE GOTHENBURG}

Rosling y cols. 1976 (19), tras evaluar la importancia de un alto nivel de higiene oral tras el tratamiento quirúrgico de la "periodontitis" (20), estudiaron la capacidad de curación del tejido periodontal y compararon los resultados del tratamiento periodontal utilizando diferentes métodos para la eliminación de la bolsa periodontal, en pacientes mantenidos bajo un estricto programa de higiene oral durante dos años.

Las técnicas utilizadas fueron (1) el colgajo de reposición apical (21) con eliminación de defectos infraóseos (AFB), (2) colgajo de reposición apical con curetage de los defectos óseos pero sin remover hueso (AF), (3) "colgajo de Widman" (22) con eliminación de defectos infraóseos (WFB), (4) "colgajo de Widman" con curetage de los defectos óseos pero sin remover hueso (WF), (5) gingivectomía (23),[24) con curetage de los defectos óseos pero sin remover hueso (G).

Se evaluó el índice de placa (IP) (25), índice gingival (IG) (26), la media individual de las profundidades de bolsas (PB) para todas las unidades gingivales (media de todas las localizaciones de todos los dientes) y para cada localización (vestibular, lingual y aproximal) en basal y a los 6, 12, 24 meses. Además se evaluaron los cambios en el nivel de hueso interproximal.

En la evaluación a 2 años, los autores encontraron que, con todas las técnicas, tanto el IP como el IG disminuyeron hasta rondar valores cercanos a cero. También la $\mathrm{PB}$ se vio reducida tras los tratamientos, aunque los valores entre basal y a los 24 meses no presentaron diferencias estadísticamente significativas. Con la gingivectomía se obtuvieron los peores resultados.

La pérdida de inserción varió en función de las localizaciones y de las técnicas utilizadas. En general, los mejores resultados se obtuvieron en zonas proximales, donde se consiguió ganancia de inserción con $\mathrm{AF}$ y WF (ligeramente mayor), y pérdida con G. Se perdió inserción en vestibular solamente tras AFB. Por lo demás no se produjeron cambios significativos.

Para los cambios en el nivel del hueso interproximal los resultados demostraron una menor reducción del hueso alveolar marginal y un mayor relleno óseo interproximal tras $A F(1,9 \mathrm{~mm}) \mathrm{YWF}(3,1 \mathrm{~mm})$ que tras los colgajos que incluían procedimientos de resección ósea.

Rosling (27), algunos años después, publica otro estudio con prácticamente la misma metodología, aunque 
esta vez no se trataron solo 50 pacientes (grupo test), sino que también se compararon con un grupo control de 25 pacientes.

Tanto el grupo test como el grupo control recibieron los tratamientos quirúrgicos (19), de manera randomizada. Pero, solamente el grupo test fue incluido en un estricto programa de terapia de mantenimiento (una vez al mes, durante dos años) mientras que los pacientes del grupo control fueron citados una vez al año para un raspado supragingival. Los pacientes del grupo control a los 2 años no fueron capaces mantener un adecuado control de placa. Hubo recurrencia de la enfermedad y hubo un progresivo desarrollo de la "periodontitis".

En la evaluación a 6 años los datos mostraron que para las bolsas iniciales de 4-6 mm, los tratamientos utilizados no proporcionaron DES entre ellos. Tampoco hubo DES en la ganancia del NI para estos tratamientos. Todavía los colgajos que no preveía cirugía ósea obtenían una mayor ganancia de inserción.

Las bolsas iniciales $>7 \mathrm{~mm}$ seguían mostrando una significativa reducción de las bolsas para todos los grupos, con los peores resultados para la gingivectomía.

No hubo en ningún caso pérdida de inserción, sino que todos los tratamientos resultaron en una ganancia de NI. Solo para los grupos AFYWF las diferencias en el NI comparado con los datos al inicio fueron ES.

En 1984, Lindhe y cols. (28) hicieron un estudio con 15 sujetos con el objeto (1) de analizar el papel del control de placa individual en la prevención de la recurrencia de la "enfermedad periodontal" y (2) evaluar el estado periodontal de estos grupos de pacientes después de 5 años tras el término del tratamiento activo, en particular en bolsas $>3 \mathrm{~mm}$.

No obstante, sus objetivos no eran exactamente evaluar las diferencias entre el tratamiento quirúrgico y el no quirúrgico, sino demostrar la importancia de la higiene oral de los pacientes (en conseguir los objetivos de la terapia). Demostraron que una tendencia a obtener mejores resultados, en cuanto a PS y NI, en las bolsas $\geq 3 \mathrm{~mm}$, en el grupo de paciente con un alto nivel de higiene oral, sin ninguna influencia (no diferencias estadísticamente significativas) por parte de los diferentes tratamientos utilizados (quirúrgico vs no quirúrgico). Según lo reportado en la discusión, "la presente investigación demostró que el nivel de higiene oral evidentemente tenía una influencia importan- te sobre los resultados a largo plazo del tratamiento de la enfermedad periodontal y sobre la recurrencia de los signos de gingivitis y la eventual ulterior pérdida de inserción o aumento de la profundidad de sondaje". Además que "el determinante crítico en la terapia periodontal no es la técnica per se que se utiliza para la eliminación de la infección subgingival, sino el desbridamiento subgingival de la superficie radicular, que debe realizarse de manera eficaz".

Por otro lado, estos mismos autores (29) revisando los estudios precedentes $(13,15,28)$ reevaluaron la importancia de la PB inicial en relación a la capacidad de desbridamiento no quirúrgico, que parece ser más complicado de realizar en bolsas más profundas y, según lo hipotetizado por estos autores, lo que pudiera ocasionar una recurrencia de la enfermedad en menos tiempo.

Los objetivos fueron (1) comparar los resultados iniciales de la terapia quirúrgica y no quirúrgica en sujetos con enfermedad periodontal avanzada y (2) determinar la incidencia de las recurrencias de la enfermedad durante el mantenimiento en sujetos que habían recibido la terapia no quirúrgica y la cirugía de acceso. Es un estudio de 12 años en 64 pacientes (se perdieron 4 sujetos en TQ y 8 en RAR) divididos en dos grupos, tratamiento quirúrgico (TQ) y raspado y alisado radicular (RAR). Se les trataron en paralelo.

Tomando como unidad de estudio el paciente (individuo) para el análisis de los datos en basal se evaluaron el número de dientes (se excluyen los molares), índice de placa (IP), el sangrado al sondaje (SS), el nivel clínico de inserción (NCI) y la profundidad de bolsa al sondaje (PBS). No hubo diferencias estadísticamente significativas entre los dos grupos al inicio. En cuanto a la pérdida de los dientes al año, había diferencias estadísticamente significativas (DES) entre los dos grupos, con mejores resultado para el grupo RAR. Siguió siendo igual durante el resto del estudio aunque no se aludió a la significación estadística.

El IP bajó durante el primer año en ambos grupos de manera similar y estadísticamente significativa (ES) manteniéndose en niveles adecuados durante todo el período de mantenimiento (inferior al $15 \%$ en todo momento). El SS se comportó de manera similar el primer año pero en las siguientes reevaluaciones los valores aumentaron significativamente en ambos grupos. La PBS se redujo de manera ES en ambos grupos en comparación al inicio. Estas diferencias fueron mayores el primer año y disminuyeron durante los siguien- 
tes, manteniéndose tales hasta los 13 años. No hubo DES entre los dos tratamiento aunque en el grupo TQ hubo en todo momento un numero significativamente mayor $(75-60 \%)$ de sitos con bolsas superficiales $(<4$ $\mathrm{mm}$ ) en comparación con el grupo RAR (60-55\%).

Las diferencias entre la media anual de la pérdida de inserción no fue ES entre los grupos y hubo las mismas variaciones para los dos grupos con respecto al inicio. Existió una tendencia a que las bolsas superficiales perdieron inserción, mientras que una ganancia se obtuvo para las bolsas más profundas. Entre el primer y tercer año tras el tratamiento activo había, en el grupo RAR, un mayor número de sujetos tratados que exhibía signos de progresión de enfermedad en comparación con el grupo TQ.

1. El CWF consigue una mayor ganancia de inserción en bolsas moderadas y un mayor relleno óseo, sobretodo en el área interproximal.

2. La terapia quirúrgica fue más efectiva que el tratamiento no quirúrgico en reducir la media total de la PBS en y en la eliminación de las bolsas profundas.

3. Entre el 1-3 año tras el tratamiento existió, un mayor porcentaje de sujetos (29\%) tratados con terapia periodontal no quirúrgica que exhibían progresión de la enfermedad en comparación con los tratados quirúrgicamente (14\%).

4. En ambos grupos, fue posible establecer una condición periodontal capaz de ser mantenida durante los años de seguimiento (12 años), con un pequeño número de episodios de recurrencia.

5. Hay menos recurrencia de enfermedad periodontal en los pacientes tratados con cirugía que los tratados con tratamiento básico.

\section{ESCUELA DE MINNESOTA}

Pihlstrom y cols. en 1983 (30) tras una atenta revisión de la literatura de estudios que comparaban los resultados obtenido con tratamiento quirúrgico y no quirúrgico de la enfermedad periodontal, reporta los datos obtenido, en un estudio con 6 años y medio de seguimiento y con el mismo objetivo. Los 17 pacientes recibieron de manera aleatoria, y con diseño a boca partida, dos tratamientos: el RAR y el RAR más CWM.

Los datos también agrupados según la profundidad de bolsa inicial fueron evaluados por la índice de placa, cálculo, inflamación gingival, PS y NI. De acuerdo con estudios precedentes $(31,16,32)$ ambos tratamientos fueron efectivos para el tratamiento de la "periodontitis". De hecho, demostraron que las dos técnicas obtuvieron, a los 6 años y medio, las mismas ganancias de inserción clínica en las bolsas profundas ( $\geq 7 \mathrm{~mm}$ ) y en bolsas moderadas, aunque en estas ultimas para el RAR solo, las ganancias fueron superiores. En las bolsas superficiales había una mayor pérdida de inserción tras el RAR + CWM que con RAR solo.

La PS era mayormente reducida durante los primeros años de tratamiento (1-3 años) tras el tratamiento quirúrgico que con el no quirúrgico, aunque estas diferencias iban disminuyendo hasta ser no significativa a los 6 años y medio, confirmando lo reportado en estudios precedentes $(16,32)$. Según los resultados de este estudio no había diferencias estadísticamente significativas en cuanto a las recurrencia de bolsas profundas entre los dos tratamientos desde los 2 hasta los 6 años y medio.

Tampoco hubo DES durante los primeros 2 años post tratamiento para la reducción de bolsa en bolsas profundas entre RAR y RAR más CWM. Sí hubo diferencia entre las dos terapias a los 6 años y medio. La reducción de bolsas fue ES en las bolsas profundas con respecto a basal solamente para RAR + CWM.

1. Ambos métodos son igual de efectivos en el tratamiento de la "periodontitis".

2. El tratamiento del surco crevicular con procedimiento de colgajo resulta en relevante pérdida del nivel clínico de inserción. Con RAR solo era posible mantener los niveles clínicos de inserción en estas "bolsas".

3. Para las bolsas de 4-6 mm ambos métodos resultan igualmente efectivos en la reducción de bolsa. Mientras que ambos métodos eran capaces de mantener el nivel clínico de inserción, una ganancia de inserción ligeramente mayor se obtuvo tras el RAR sólo que con RAR más CWM.

4. Para las bolsas de $\geq 7 \mathrm{~mm}$ el procedimiento a colgajo resultaba mantener la reducción de bolsas hasta los 6 años y medio mientras que RAR solo conseguía este resultado hasta los 3 años. No hubo DES entre las ganancias en lo niveles de inserción que se obtuvieron con los dos tratamientos.

\section{ESCUELA DE WASHINGTON}

Townsend-Olsen y cols. en el 1985 (33) trataron de evaluar en 12 pacientes con "enfermedad periodontal moderada" si había alguna diferencia entre el trata- 
miento con curetage a cielo abierto con (con CO) o sin cirugía ósea ( $\sin \mathrm{CO}$ ). Estos pacientes afectados de forma simétrica por la enfermedad presentaban una profundidad de sondaje media de $4 \mathrm{~mm}$ con un rango que iba de 1 a $8 \mathrm{~mm}$.

En la evaluación del índice de placa e inflamación gingival no encontraron diferencias estadísticamente significativas entre las dos intervenciones ni en basal ni durante, ni al final del estudio. Se evaluaron también los cambios en la anchura de la encía queratinizada, la cual con ambas técnicas y sin diferencias estadísticamente significativas, fue aumentada. A pesar de una mayor frecuencia de las recesiones de los tejidos gingivales tras el tratamiento con cirugía ósea (con CO), no hubo ninguna diferencia estadísticamente significativa en los NI entre los dos procedimiento. Además se obtuvieron mejores resultados también en cuanto a la disminución de la profundidad de sondaje en la zona interproximal tras la CO (PS residual $=3,1$ con CO; PS residual $=3,4 \sin \mathrm{CO}$ ). Aunque eso no fue cierto en vestibular y lingual.

Las áreas tratadas con CO presentaban sólo el 18\% de localizaciones con PS $\geq 4 \mathrm{~mm}$ y sangrado al sondaje, frente al $42 \%$ de los casos sin CO. Además se conservó una altura de la cresta adecuada sin que a los 5 años hubiera diferencias entre ambas técnicas.

1. Aunque a los 6 meses con ambas técnicas se redujeron las bolsas, a los 5 años sólo mantuvieron una disminución significativa en aquellas zonas tratadas con $\mathrm{CO}$.

2. A los 5 años, hay 2,3 veces más localizaciones con $\mathrm{PS} \geq 4 \mathrm{~mm}$ y con sangrado al sondaje en áreas en las que no se realizó $\mathrm{CO}$.

3. EL margen gingival se situó más apical con $\mathrm{CO}$ en todos los intervalos evaluados.

4. La CO es más efectiva a la hora de reducir las bolsas, en concreto, para las bolsas de 5-8 $\mathrm{mm}$ preoperatorio.

5. Los datos del estudio muestran una tendencia a la recidiva en zonas tratadas $\sin \mathrm{CO}$.

\section{ESCUELA DE HOUSTON}

En el 2001 Becker W y cols. (34) evaluaron los resultados de un estudio de 5 años sobre la efectividad de los diferentes tratamientos periodontales a la hora de reducir la profundidad de sondaje y de mantener los niveles clínicos de inserción. Tomando como unidad de estudio el paciente, se compararon el RAR, cirugía ósea (CO) y colgajo de Widman modificado (CWM) a 5 años de mantenimiento.

Aunque el estudio se terminó a los 12 años solo se reportaron los resultados a los 5 años por disminución del número de pacientes al final del estudio. Se evaluaron los cambios en el IP, IG, PS estratificados según la severidad de las bolsas iniciales (1-3 mm, 4-6 mm, $\geq$ $7 \mathrm{~mm}$ ) y NCI también según una distinta categoría (0$2 \mathrm{~mm}, 3-5 \mathrm{~mm}, \geq 6 \mathrm{~mm}$ ).

De acuerdo con otros autores, en las bolsas poco profundas hubo un ligero aumento de la PS (no estadísticamente significativa) a los 5 años para los tres tratamientos y un aumento de la pérdida del nivel de inserción (estadísticamente significativo) respecto al inicio para los tres tratamientos.

En las bolsas moderadas (4-6 mm) se obtuvieron mejores resultados para CO desde los 2 a los 4 años con respecto a RAR. No hubo diferencias entre los tratamientos a los 5 años tanto para la PS como para el NI. La progresión de los resultados fue el mismo para las bolsas profundas ( $\geq 7 \mathrm{~mm}$ ) y a los 5 años la media de las profundidades de bolsa era para RAR 6.17; 4.87 para CO y 4.66 para CWM. La media para el NI era $6,00 \mathrm{~mm}$ para RAR, 5, 11 para CO y 5,77 para CWM.

A los 5 años el grupo CO tenía el $72 \%$ de bolsas poco profundas, el $22,1 \%$ de bolsas moderadas y el $4,6 \%$ de bolsas profundas. El grupo CWM tenía respectivamente el $72,7 \%$; el $21,8 \%$ y el 5,6\%. El grupo RAR tenía los valores eran similares a los de la cirugía ósea, es decir $73,2 \% ; 23,4 \%$ y $3,4 \%$.

Se reportaron los datos relativos a las recesiones que hubo para los tres tratamientos. A los 5 años no hubo diferencias estadísticamente significativas entre las tres modalidades. Solo se notaba una mayor estabilidad de los tejidos, tras los 5 años con respecto a basal, para el grupo de RAR (91,1\% de lugares sin cambios).

Los autores reconocen como limitaciones de su estudio la falta de tests con mayor potencia estadística y la falta de una muestra mayor de pacientes (solamente 16 pacientes) aunque se (1) demuestre que la terapia periodontal es efectiva y que (2) la higiene oral personal junto con el mantenimiento por parte del profesional pueden conservar la salud periodontal con una mínima pérdida dentaria. De 229 dientes, uno se perdió por fractura radicular a causa de una complicación de la endodoncia y 6 por causas desconocidas. 
1. La terapia dental es efectiva para la curación de la "enfermedad periodontal".

2. La higiene oral personal junto con el mantenimiento por parte del profesional pueden conservar la salud periodontal con una mínima pérdida dentaria.

3. RAR, CO y CWM fueron efectivos en reducir la PS con ligeros cambios en nivel clínico de inserción.

4. En circunstancia apropiada, cada uno de los procedimientos puede ser efectivo.

5. La decisión del tratamiento varía de acuerdo con el diagnóstico y el plan de tratamiento del paciente.

\section{ESCUELA DE NEBRASKA}

Kaldahl y cols. (35) justificaron su estudio con la necesidad de resultados proveniente de estudios clínicos, a largo plazo, que compararan diferentes tipo de tratamientos y en un mayor número de pacientes (82).

Los objetivos fueron entonces comparar (1) los efectos clínicos que producen 4 tipos de tratamiento periodontales y (2) cómo estos efectos se mantengan tras 7 años de seguimiento (sólo terminaron el estudio 51 pacientes).

Cada paciente recibió en cada cuadrante uno de los cuatro tratamientos: tartrectomía supragingival (TS), RAR, CWM y RAR a cielo abierto más cirugía ósea (CO).

La unidad primaria de análisis fue el paciente, se calculó la media de los cambios de cada parámetro cuantitativo en cada paciente y los datos fueron agrupados en base a la severidad de la PS (1-4 mm bolsas poco profundas; 5-6 mm bolsas moderadas; $\geq 7 \mathrm{~mm}$ bolsas profundas).

Los tratamientos se asignaron de manera aleatoria y se hicieron en dos fases de manera que en la primera, los 4 cuadrantes recibieron el TS y después tres de los cuatro (los designados para RAR, CWM y CO) recibían el RAR. En la segunda fase se efectuaba CWM y CO a los dos cuadrantes asignados para el tratamiento quirúrgico. El criterio de inclusión para la realización de las cirugías era la presencia de por lo menos una bolsa $\geq 5 \mathrm{~mm}$ tras la fase I. La región anterosuperior fue excluida por razones estéticas. Cada 3 meses se efectuaba la terapia de mantenimiento (Instrucción de higiene oral, TS, RAR de las bolsas restantes).

Se tomaron como iniciales los registros (1 examinador, ciego) en la primera visita (Examen 1), a las 4 sema- nas tras la fase I (Examen 2), 10 semanas después de la fase II (Examen 3) y anualmente antes del mantenimiento periodontal. A los 2 años los dientes que solo se trataron con TS, al necesitar retratamiento (RAR) por pérdida de $\mathrm{NI} \geq 3 \mathrm{~mm}$ al examen 1 , se excluyeron del estudio, mientras que para los que tras RAR, CWM y CO perdieron NI $\geq 3 \mathrm{~mm}$ al examen 3 se les realizó RAR y se mantuvieron para el examen estadístico.

Los resultados sugieren que el TS era capaz de provocar cambios positivos (probablemente por alteración de la placa subgingival, respuesta sistémica a los otros tratamientos periodontales, etc.) en cuanto a la PS, el $\mathrm{NI}$, las recesiones y a la reducción de los signos de la inflamación de manera ES con respecto a basal aunque la significación estadística no se mantuvo durante todo el estudio.

Tomando como, iniciales, las mediciones hechas en la primera visita (examen 1) los autores encuentran que tanto el RAR como el CWM y el CO redujeron la PS de manera ES con respecto a basal en todas las categorías de bolsas. Para las bolsas moderadas y profundas CO mostró mejores resultados respecto a CWM y RAR aunque no existieron diferencias estadísticamente significativas entre los diferentes tratamientos a partir de los 3 y 5 años para las dos categorías de bolsas respectivamente.

Con los cuatro tratamientos se producía una ganancia de los NI en todas las categorías de bolsas, menos para $\mathrm{CO}$ que en las bolsas poco profundas perdió inserción. En las bolsas moderadas los resultados obtenidos con RAR eran estadísticamente mejores en comparación con los reportados para CO. El CWM presentaba valores intermedios siendo no estadísticamente significativos. En bolsas profundas no hubo diferencias entre los tratamientos.

Cuando se compararon los resultados tomando como basal las mediciones correspondientes al examen 3 resultó que las bolsas poco profundas fueron sujetas a un incremento de la profundidad y a una pequeña pérdida en el NI.

La bolsas moderadas y profundas se redujeron durante el mantenimiento y mostraron una tendencia a la ganancia en el NI con los tres tratamientos con excepción de CO con la que no se ganó inserción en las bolsas $\geq 7 \mathrm{~mm}$.

1. Para las reducciones de bolsas, las bolsas moderadas y profundas CO mostró mejores resultados res- 
pecto a CWM y RAR aunque no existieron diferencias estadísticamente significativas entre los diferentes tratamientos a partir de los 3 y 5 años para las dos categorías de bolsas respectivamente.

2. Para la ganancia de inserción, con los cuatro tratamiento se producía una ganancia de los NI en todas las categorías de bolsas, menos para $\mathrm{CO}$ que en las bolsas poco profundas perdió inserción. No hubo diferencias entre los tratamientos.

3. Para la ganancia de inserción, en bolsas moderadas los resultados obtenidos con RAR eran estadísticamente mejores en comparación con los reportados para CO. El CWM presentaba valores intermedios no siendo estadísticamente significativos.

4. El sólo TS no era suficiente para mantener el estado de salud. A los 2 años los dientes que solo se trataron con TS necesitaron retratamiento (RAR) por manifestar, al examen 1 , pérdida de $\mathrm{NI} \geq 3 \mathrm{~mm}$.

\section{DISCUSIÓN}

Desde el principio de los años 70 hubo la necesidad de evaluar a largo plazo los efectos de los diferentes tratamientos periodontales con la idea de aclarar sus beneficios, sus necesidades y sus dudas. Las conclusiones reportadas en cada estudio resultan ser en muchas ocasiones discordantes. Probablemente debido a las diferencias en el diseño del estudio, en la metodología de la recogida de los datos, de la presentación de los resultados, de los criterios de inclusión utilizados, como también por utilizar técnicas de randomización diferentes y por estudiar poblaciones distintas (etc...) estas conclusiones, sobre todo desde el punto de vista clínico, pueden resultar equívocas. Faltando entonces de validez externa las conclusiones arriba citadas no pueden asumirse como líneas guías, en el acto de elegir uno u otro tratamiento.

Un esfuerzo en tal dirección, se hizo mediante la ejecución de una revisión sistemática. Heitz-Mayfield L. J. y cols en 2002 (2) tras una atenta selección de los ensayos clínicos presentes en la literatura y tras haber descartado la mayoría de éstos (solo quedaron 6) por no cumplir con los criterios de inclusión, presentó los resultados de su metaanálisis con los datos resultantes al primer año de seguimiento. Los datos se presentaron en relación a la severidad de la PS inicial (32).

Se reportaron las diferencias de las medias ponderadas por cada parámetro. Para las bolsas de clase I (1-3 $\mathrm{mm})$, había una menor pérdida de inserción $(0,5 \mathrm{~mm})$ tras el RAR que con el tratamiento quirúrgico (TQ), mientras que no fue posible una disminución de la profundidad de sondaje tras los dos tratamientos $(15,32$, $30,36)$.

En la clase II (4-6 mm) de bolsas iniciales, los resultados fueron favorables para $\operatorname{RAR}(15,32,30,36)$ en cuanto a los cambios en el NI (menor pérdida de NI de 0,4 $\mathrm{mm}$ ) y una mayor reducción de la profundidad de sondaje $(0,2 \mathrm{~mm})$ tras el tratamiento quirúrgico. $(15,30)$. Para las bolsas de clase III (> $6 \mathrm{~mm}$ ) los resultados fueron más favorables con el tratamiento quirúrgico tanto en la reducción de la PS $(15,30)$ resultando mayor de $0,58 \mathrm{~mm}$, como para la ganancia en el NI que era mayor de $0,2 \mathrm{~mm}(15,30,32,36)$. A causa de los estrictos criterios de inclusión adoptados, el número de estudios incluidos en el metaanálisis fue bastante reducido.

Aunque se trate del único metaanálisis hoy en día disponible sobre este tema, no significa que estos datos sean la panacea para la resolución a nuestras dudas. Como también los mismos autores lamentan, no fue posible efectuar una evaluación de los datos a más largo plazo mediante el metaanálisis, primero porque había diferencias en los diferentes términos utilizados para describir la población estudiada, la severidad de la enfermedad (Tabla 1) y en el rango de edad de dichas poblaciones. Segundo, porque había discordancias también en las terapias iniciales como también en las terapias periodontales de soporte efectuadas (Tabla 1), de hecho, en algunos estudios se efectuaba un tratamiento de mantenimiento una vez al mes durante 2 años $(19,27)$ otros se le incluía en un programa estricto de mantenimiento durante los primeros meses $(15,28)$ y después se citaban los pacientes cada tres meses. Mientras en otros solo se realizó terapia de mantenimiento cada 3 meses $(37,29,30,35)$ o cada 6 meses (33). Se resaltó, la necesidad de ulteriores ensayos clínicos randomizados que respetaran los principios metodológicos comunicados por el CONSORT.

No obstante, las diferencias en cuanto concierne al primer año de seguimiento, los datos relativos a las bolsas superficiales (1-3 mm) tienden y las bolsas profundas $(>6 \mathrm{~mm}$ ) resultaron ser representativos de los reportados en cada uno de los siete estudios evaluados a largo plazo. Esto no ocurrió para las bolsas moderadas donde según los autores (15) de la escuela de Michigan había una mayor reducción de bolsas con el CWM mientras que según los autores (30) de la escuela del Minnesota no había diferencias. Los dos (15, 30) concordaban, junto con los autores (32) de la escuela de Suecia, sobre una mayor ganancia de inser- 


\begin{tabular}{|c|c|c|c|c|c|c|c|c|}
\hline \multicolumn{9}{|c|}{ TABLA 1} \\
\hline & \multirow[t]{2}{*}{ Autores } & \multirow[t]{2}{*}{ Mantenimiento } & \multirow{2}{*}{$\begin{array}{l}\text { Dientes } \\
\text { incluidos }\end{array}$} & \multirow{2}{*}{$\begin{array}{l}\text { Criterios de } \\
\text { inclusión }\end{array}$} & \multicolumn{2}{|c|}{ BI 4-6mm } & \multicolumn{2}{|c|}{$\mathrm{BI} \geq 7 \mathrm{~mm}$} \\
\hline & & & & & PS & $\mathbf{N I}$ & PS & NI \\
\hline Michigan & $\begin{array}{l}\text { Knowles } \\
1979\end{array}$ & Cada 3 meses & Boca partida & $\begin{array}{l}\text { Más de } 1 \text { bolsa } \\
\geq 4 \mathrm{~mm}\end{array}$ & $Q x$ & ND & ND & Qx \\
\hline Michigan & $\begin{array}{l}\text { Ramfjord } \\
1987\end{array}$ & $\begin{array}{l}\text { Cada semana } \\
\text { durante un mes y } \\
\text { cada } 3 \text { meses } \\
\text { durante } 5 \text { años }\end{array}$ & Boca partida & $\begin{array}{l}\text { Más de } 1 \text { bolsa } \\
\geq 4 \text { mm y con, } \\
\text { por lo menos, } \\
20 \text { dientes }\end{array}$ & $Q x$ & NQx & ND & ND \\
\hline Gothenburg & $\begin{array}{l}\text { Rosling } \\
\text { 1976/83 }\end{array}$ & $\begin{array}{l}\text { Cada } 2 \text { semanas } \\
\text { durante } 2 \text { años }\end{array}$ & $\begin{array}{l}\text { Incisivos, } \\
\text { premolares } \\
\text { y superficies } \\
\text { mesiales de } \\
\text { los molares }\end{array}$ & Bolsas $\geq 3 \mathrm{~mm}$ & ND & ND & Qx & Qx \\
\hline Gothenburg & $\begin{array}{l}\text { Lindhe } \\
1984\end{array}$ & $\begin{array}{l}\text { Cada } 2 \text { semanas } \\
\text { durante } 6 \text { meses. } \\
\text { Cada } 3 \text { meses entre } \\
6-24 \text { meses. } \\
\text { Cada } 4-6 \text { meses } \\
\text { entre } 25-60 \text { meses }\end{array}$ & Boca partida & Bolsas $>3 \mathrm{~mm}$ & ND & $\mathrm{ND}$ & ND & ND \\
\hline Gothenburg & $\begin{array}{l}\text { Serino } \\
2001\end{array}$ & $\begin{array}{l}\text { 3-4 veces al año y los } \\
\text { sitios con } P P D \geq 5 \mathrm{~mm} \\
\text { se le raspaba } \\
\text { nuevamente }\end{array}$ & No molares & $\begin{array}{l}\text { Mínimo } 12 \\
\text { dientes no } \\
\text { molares con } \\
\mathrm{PS} \geq 6 \mathrm{~mm} \text { y } \\
\mathrm{PO} \geq 6 \mathrm{~mm}\end{array}$ & ND & ND & ND & ND \\
\hline Minnesota & $\begin{array}{l}\text { Pihlstrom } \\
1983\end{array}$ & Cada 3-4 meses & Boca partida & $\begin{array}{l}\text { Distribución } \\
\text { bilateral de } \\
\text { bolsas de } \\
\text { l a } 14 \mathrm{~mm}\end{array}$ & ND & NQx & $Q x$ & ND \\
\hline Washington & $\begin{array}{l}\text { Townsend- } \\
\text { Olsen } \\
1985\end{array}$ & $\begin{array}{l}\text { Cada } 6 \text { meses a } \\
\text { partir del } 6 \text { mes } \\
\text { y durante } 2 \text { años }\end{array}$ & $\begin{array}{l}\text { Molares y } \\
\text { premolares }\end{array}$ & $\begin{array}{l}\text { PB variable } \\
\text { entre } 1 \text { y } 8 \mathrm{~mm} \text {, } \\
\text { es decir } 4 \mathrm{~mm} \\
\text { de media }\end{array}$ & Qx & $Q x$ & $Q x$ & Qx \\
\hline Houston & $\begin{array}{l}\text { Becker } \\
2001\end{array}$ & $\begin{array}{l}\text { Visita de control } \\
\text { cada semana durante } \\
6 \text { meses y después } \\
\text { mantenimiento cada } \\
3 \text { meses }\end{array}$ & Boca partida & $\begin{array}{l}2 \text { o más sitio con } \\
\text { PI } \geq 6 \text { mm en los } \\
\text { sextantes } \\
\text { posteriores ( } 46)\end{array}$ & ND & ND & ND & ND \\
\hline Nebraska & $\begin{array}{l}\text { Kaldahl } \\
1996\end{array}$ & Cada 3 meses & Boca partida & $\begin{array}{l}\text { Para todos los } \\
\text { dientes TS y RAR. } \\
\text { CO y CWM para } \\
\text { los dientes con } \\
\text { PS } \geq 5 \mathrm{~mm}\end{array}$ & $\begin{array}{c}\mathrm{Qx} \\
(\mathrm{CO}, \mathrm{CW})\end{array}$ & $\mathrm{NQx}$ & $\begin{array}{c}\mathrm{Qx} \\
(\mathrm{CO}, \mathrm{CW})\end{array}$ & ND \\
\hline
\end{tabular}

BI: bolsas iniciales; Qx: tratamiento quirúrgico; NQx: tratamiento no quirúrgico; ND: no diferencia entre los tratamiento; CO: cirugía ósea; CWM: colgajo de Widman modificado; Ts: Desbridamiento supragingival; Ps: profundidad de sondaje; PB: profundidad de bolsa; PI: pçerdida de inserción; PO: pérdida ósea 
ción tras el tratamiento no quirúrgico para las bolsas con esta profundidad inicial mientras que en el siguiente estudio presentado por este último grupo (36) no hubo diferencias entre los tratamientos.

Otro aspecto de no menos importancia que no se ha podido aclarar en esta revisión sistemática es la evaluación de la necesidad del tratamiento quirúrgico sin o con remodelado óseo. Según algunos autores (33), se obtienen mejores resultados haciendo cirugía ósea que sin ella, en cuanto a la reducción de la PS y en la disminución de la probabilidad de recidiva en las zonas tratadas con CO. Otros autores (34) demuestra que tras 5 años y medio de tratamiento no existieron DES entre los tres tratamientos (RAR, CWM y CO) en la reducción de la PS y la ganancia del NI. Lo mismo se observó para la recesión de los tejidos gingivales. Estos datos fueron confirmados por Kaldahl y cols. (35) adjuntando también que 7 años tras los tratamientos (TS, RAR, CWM y CO) solamente con CO no se logró ganar NI, mientras que no hubo diferencias en la reducción de la PS.

\section{CONCLUSIONES}

Según la interpretación de los datos presentados en los estudios revisados, quedan por aclararse algunos conceptos que todavía no poseen sostén en la literatura científica.

\section{A CORTO PLAZO}

La revisión sistemática de Heitz-Mayfield L. J. y cols en 2002 (2) nos ofrece las mejores respuestas a nuestras preguntas.

- Para las bolsas de clase I (1-3 mm), había una menor pérdida de inserción $(0,5 \mathrm{~mm})$ tras el RAR que con el tratamiento quirúrgico (TQ), mientras que no fue posible una disminución de la profundidad de sondaje tras los dos tratamientos

- Para las bolsas de clase II (4-6 mm), los resultados fueron favorables para RAR en cuanto a los cambios en el NI (menor pérdida de NI de 0,4 mm) y una mayor reducción de la profundidad de sondaje $(0,2 \mathrm{~mm})$ tras el tratamiento quirúrgico.

- Para las bolsas de clase III (> $6 \mathrm{~mm}$ ), los resultados fueron más favorables con el tratamiento quirúrgico tanto en la reducción de la PS resultando mayor de $0,58 \mathrm{~mm}$ como para la ganancia en el NI que era mayor de $0,2 \mathrm{~mm}$.

\section{A LARGO PLAZO}

- Para las bolsas de clase I (1-3 mm), no es posible una disminución de la profundidad de sondaje con ambos tratamientos mientras que con RAR se produce una menor pérdida de inserción.

- Para las bolsas de clase II (4-6 mm), el tratamiento quirúrgico, según algunos autores, obtiene mejores resultados en cuanto a la reducción de la PS (13, $15,33,35)$ y en la ganancia del NI (33). Mientras que otra franja acertó que no hay DES entre los tratamientos para la reducción de la PS $(19,27,28,29$, 30,34 y para la ganancia en el NI $(13,27-29,34)$. Finalmente según $(15,30,35)$ el tratamiento no quirúrgico fuera capaz de obtener mejores resultados en cuanto a la ganancia del NI (Tabla 1).

- Para las bolsas de clase III (> $6 \mathrm{~mm}$ ), la tendencia de algunos estudios para la reducción de la PS es la de igualar los resultados a largo plazo $(13,15,28$, $38,34)$ mientras que otros aciertan la mayor eficacia del tratamiento quirúrgico $(27,30,33,35)$. Para la ganancia del NI no hubo DES según algunos autores $(15,28,30,34,35,38)$ mientras según otros hubo mejores resultado con el tratamiento quirúrgico $(13,27,33)$ (Tabla 1).

- Parece que la técnica terapéutica pierde valor, dando más importancia al mantenimiento.

Al parecer, la decisión de la técnica a utilizar queda en manos del clínico. Puede que la clave esté en individualizar los casos y en buscar objetivos terapéuticos. Es decir, en aquellos casos en los que el acceso sea complicado, una técnica quirúrgica sería la mejor opción. Un claro ejemplo es la afectación furcal. Autores como Fleischer y Matia $(39,40)$ demostraron la mayor eficacia en la eliminación de cálculo al utilizar una técnica quirúrgica versus una no quirúrgica.

Otros estudios en los que se evaluó la eficacia de diferentes tratamientos quirúrgico sobre dientes multirradiculares con afectación furcal pueden ayudarnos decantarnos hacia este tipo de tratamiento (41-45). Es obvio que si nos planteamos cualquier tipo de procedimiento regenerativo, la opción de tratamiento será quirúrgica.

En resumen, podríamos decir que las ventajas del tratamiento quirúrgico radican fundamentalmente en el acceso. Si somos capaces de acceder a todas las localizaciones con RAR y las reevaluaciones son positivas, este tratamiento no quirúrgico puede ser una opción. Pero en aquellas localizaciones en las que nuestro acceso sea insuficiente, debemos plantearnos un tratamiento quirúrgico; ya sea en bolsas muy profundas 
poco accesibles, dientes multirradiculares con afectación furcal o defectos óseos candidatos o no a regeneración periodontal. Por supuesto que todos ello debe estar acompañado por un buen mantenimiento periodontal ya que, como demuestra la literatura, es la clave de nuestro tratamiento y futuro éxito terapéutico.

\section{SUMIMARY}

Periodontal diseases are bacterial infections that destroy the attachment fibres and supporting bone that hold the teeth in the mouth. Left untreated, these diseases can lead to tooth loss (Medical Dictionary). Longitudinal studies centred their attention on chronic periodontitis. It has been documented the decisive role played by microbiological plaque in the initiation of gingivitis and that, the harmful effect on the tissues and its severity, are controlled by the complex host-parasite interaction. Treatment of periodontal lesion can be carried out either by non-surgical treatment, to eliminate and prevent the recurrence of bacterial deposits, or by surgical treatment, to create access for professional debridment of infected root surface and establish adequate gingival morphology to facilitate self plaque control. Different techniques are used to achieve the objective to improve teeth long term prognosis. Unfortunately no many studies have been able to demonstrate the effectiveness of the used technique in a long term intervals leaving unclear some questions.

\section{KEY WORDS}

Periodontal disease/therapy, non-surgical therapy, surgical therapy, systematic review.

\section{BIBLIOGRAFÍA}

1. Armitage GC. Development of a classification system for periodontal diseases and conditions. Annals of periodontology / the American Academy of Periodontology. 1999 Dec;4(1):1-6.

2. Heitz-Mayfield LJ, Trombelli L, Heitz F, Needleman I, Moles D. A systematic review of the effect of surgical debridement vs non-surgical debridement for the treatment of chronic periodontitis. Journal of clinical periodontology. 2002;29 Suppl 3:92-102; discussion 60-2.

3. Socransky SS. Relationship of bacteria to the etiology of periodontal disease. Journal of dental research. 1970 Mar-Apr;49(2):203-22.
4. Albandar JM. A 6-year study on the pattern of periodontal disease progression. Journal of clinical periodontology. 1990 Aug;17(7 Pt 1):467-71.

5. Slots J, Rams TE. Antibiotics in periodontal therapy: advantages and disadvantages. Journal of clinical periodontology. 1990 Aug;17(7 Pt 2):479-93.

6. Asikainen S, Lai CH, Alaluusua S, Slots J. Distribution of Actinobacillus actinomycetemcomitans serotypes in periodontal health and disease. Oral microbiology and immunology. 1991 Apr;6(2):115-8.

7. Ebersole JL, Cappelli D. Gingival crevicular fluid antibody to Actinobacillus actinomycetemcomitans in periodontal disease. Oral microbiology and immunology. 1994 Dec;9(6):335-44.

8. Listgarten MA, Wong MY, Lai CH. Detection of Actinobacillus actinomycetemcomitans, Porphyromonas gingivalis, and Bacteroides forsythus in an A. actinomycetemcomitans-positive patient population.J Periodontol. 1995 Feb;66(2):158-64.

9. Seymour GJ. Importance of the host response in the periodontium. Journal of clinical periodontology. 1991 Jul; 18(6):421-6.

10. Badersten A, Nilveus R, Egelberg J. Scores of plaque, bleeding, suppuration and probing depth to predict probing attachment loss. 5 years of observation following nonsurgical periodontal therapy. Journal of clinical periodontology. 1990 Feb;17(2):102-7.

11. Claffey N, Egelberg J. Clinical indicators of probing attachment loss following initial periodontal treatment in advanced periodontitis patients. Journal of clinical periodontology. 1995 Sep;22(9):690-6.

12. Ramfjord SP, Knowles JW, Nissle RR, Shick RA, Burgett FG. Longitudinal study of periodontal therapy. J Periodontol. 1973 Feb;44(2):66-77.

13. Knowles JW, Burgett FG, Nissle RR, Shick RA, Morrison EC, Ramfjord SP. Results of periodontal treatment related to pocket depth and attachment level. Eight years. J Periodontol. 1979 May;50(5):225-33.

14. Ramfjord SP, Nissle RR. The modified widman flap. J Periodontol. 1974 Aug;45(8):601-7.

15. Ramfjord SP, Caffesse RG, Morrison EC, Hill RW, Kerry GJ, Appleberry EA, et al. 4 modalities of periodontal 
treatment compared over 5 years. Journal of clinical periodontology. $1987 \mathrm{Sep}$; 14(8):445-52.

16. Hill RW, Ramfjord SP, Morrison EC, Appleberry EA, Caffesse RG, Kerry GJ, et al. Four types of periodontal treatment compared over two years.J Periodontol. 1981 Nov;52(11):655-62.

17. Prichard JF. Advances periodontal disease, 2nd edition. Philadelphia, WB Saunders Co 1972.

18. Ramfjord SPA, M. M. . Periodontology and periodontics. Philadelphia Saunders Co. 1979.

19. Rosling B, Nyman S, Lindhe J, Jern B. The healing potential of the periodontal tissues following different techniques of periodontal surgery in plaque-free dentitions. A 2-year clinical study. Journal of clinical periodontology. 1976 Nov;3(4):233-50.

20. Lindhe J, Nyman S. The effect of plaque control and surgical pocket elimination on the establishment and maintenance of periodontal health. A longitudinal study of periodontal therapy in cases of advanced disease. Journal of clinical periodontology. 1975 Apr;2(2):67-79.

21. Friedman N. Mucogingival surgery: the apically repositioned flap. J Periodontol. 1962;33:328.

22. Widman L. The operative treatment of pyorrhea alveolaris. A new surgical method. Svensk Tandlakaretidsskrift. 1917;10:85.

23. Goldman HM. Gengivectomy: indications, contraindications and methods. Am J Orthodont Oral Surg. 1964;32:33.

24. Bjorn H. Kirurgisk behandling vid parodontitis marginalis. Sveriges Tandlakare-Forbunds Tidning. 1967 $60: 942$

25. Silness J, Loee H. Periodontal Disease in Pregnancy. Ii. Correlation between Oral Hygiene and Periodontal Condtion. Acta odontologica Scandinavica. 1964 Feb;22:121-35.

26. Loee H, Silness J. Periodontal Disease in Pregnancy. I. Prevalence and Severity. Acta odontologica Scandinavica. 1963 Dec;21:533-51.

27. Rosling B. Periodontally treated dentitions: their maintenance and prognosis. International dental journal. 1983 Jun;33(2):147-51.
28. Lindhe J, Westfelt E, Nyman S, Socransky SS, Haffajee $A D$. Long-term effect of surgical/non-surgical treatment of periodontal disease. Journal of clinical periodontology. 1984 Aug; 1 1(7):448-58.

29. Serino G, Rosling B, Ramberg P, Socransky SS, Lindhe J. Initial outcome and long-term effect of surgical and nonsurgical treatment of advanced periodontal disease. Journal of clinical periodontology. 2001 Oct;28 (10):910-6.

30. Pihlstrom BL, McHugh RB, Oliphant TH, Ortiz-Campos C. Comparison of surgical and nonsurgical treatment of periodontal disease. A review of current studies and additional results after $61 / 2$ years. Journal of clinical periodontology. $1983 \mathrm{Sep} ; 10(5): 524-41$.

31. Pihlstrom BL, Ortiz-Campos C, McHugh RB. A randomized four-years study of periodontal therapy. J Periodontol. 1981 May;52(5):227-42.

32. Lindhe J, Westfelt E, Nyman S, Socransky SS, Heijl L, Bratthall G. Healing following surgical/non-surgical treatment of periodontal disease. A clinical study. Journal of clinical periodontology. 1982 Mar;9(2):115-28.

33. Townsend-Olsen C AW, van Belle G. . Alongitudinal study comparing apically repositioned flaps with and withoutosseous surgery. . The International journal of periodontics \& restorative dentistry. 1985;5(4):11-33.

34. Becker W, Becker BE, Caffesse R, Kerry G, Ochsenbein C, Morrison E, et al.A longitudinal study comparing scaling, osseous surgery, and modifiedWidman procedures: results after 5 years. J Periodontol. 2001 Dec; 72(12):1675-84.

35. Kaldahl WB, Kalkwarf KL, Patil KD, Molvar MP, Dyer JK. Long-term evaluation of periodontal therapy: I. Response to 4 therapeutic modalities. J Periodontol. 1996 Feb;67 (2):93-102.

36. Lindhe J, Nyman S. Scaling and granulation tissue removal in periodontal therapy. Journal of clinical periodontology. 1985 May;12(5):374-88.

37. Ramfjord SP, Knowles JW, Nissle RR, Burgett FG, Shick RA. Results following three modalities of periodontal therapy. J Periodontol. 1975 Sep;46(9):522-6.

38. Rosling B, Serino G, Hellstrom MK, Socransky SS, Lindhe J. Longitudinal periodontal tissue alterations during supportive therapy. Findings from subjects with normal and high susceptibility to periodontal disease. Journal of clinical periodontology. 2001 Mar;28(3):241-9. 
39. Fleischer HC, Mellonig JT, Brayer WK, Gray JL, Barnett JD. Scaling and root planing efficacy in multirooted teeth. J Periodontol. 1989 Jul;60(7):402-9.

40. Matia JI, Bissada NF, Maybury JE, Ricchetti P. Efficiency of scaling of the molar furcation area with and without surgical access. The International journal of periodontics $\&$ restorative dentistry. 1986;6(6):24-35.

41. Hamp SE, Nyman S, Lindhe J. Periodontal treatment of multirooted teeth. Results after 5 years. Journal of clinical periodontology. 1975 Aug;2(3):126-35.

42. Ross IF, Thompson RH,Jr. A long term study of root retention in the treatment of maxillary molars with furcation involvement. J Periodontol. 1978 May;49(5): 238-44.

43. Langer B, Stein SD, Wagenberg B. An evaluation of root resections. A ten-year study. J Periodontol. 1981 Dec;52 (12):719-22.

44. Carnevale G, Di Febo G, Tonelli MP, Marin C, Fuzzi M. A retrospective analysis of the periodontal-prosthetic treatment of molars with interradicular lesions. The International journal of periodontics \& restorative dentistry. 1991;11(3):189-205.

45. Svardstrom G, Wennstrom JL. Periodontal treatment decisions for molars: an analysis of influencing factors and long-term outcome. J Periodontol. 2000 Apr;71(4): 579-85.

46. Becker W, Becker BE, Ochsenbein C, Kerry G, Caffesse R, Morrison EC, et al. A longitudinal study comparing scaling, osseous surgery and modified Widman procedures. Results after one year. J Periodontol. 1988 Jun; 59(6):351-65.

\section{CORRESPONDENCIA}

\section{S. Fabrizi}

Federico Carlos Sainz de Robles, 3 bajo izq 28035 Madrid, España

Teléfono: (0034) 696872110 - 913163264

Correo electrónico: simone.fabrizi@hotmail.com 